\title{
Calculation of a two-phase system of a liquified hydrocarbon gas
}

\author{
Anna Malysheva ${ }^{1 *}$ \\ ${ }^{1}$ National Research Moscow State University of Civil Engineering, 129337, 26, Yaroslavskoe shosse, \\ Moscow, Russia
}

\begin{abstract}
Liquefied hydrocarbon gases are a mixture of chemical compounds, consisting mainly of hydrogen and carbon with different molecular structures. The main components of liquefied hydrocarbon gases are propane and butane, containing lighter hydrocarbons (methane and ethane) and heavier ones (pentane) in the form of impurities. All of these components are saturated hydrocarbons. The composition of liquefied hydrocarbon gases can also include unsaturated hydrocarbons: ethylene, propylene, butylene. Butane-butylenes may be present as isomeric compounds (isobutane and isobutylene). When designing and operating liquefied petroleum gas systems, it is necessary to take into account the external equilibrium between the liquid and the gas. The paper analyzes the change in the ratio of components, in which the relative content of lighter hydrocarbons will decrease, while the content of heavier ones will increase. With an intensive flow of gas, the temperature of the liquid will drop sharply, estimation will be disturbed, and the vessel will freeze. Therefore, when operating balloon installations, it is very important to consider safety issues that are associated with containers' filling, as well as with the change in the composition of hydrocarbon gases mixture. The discussed issues related to the calculation of a two-phase system of liquefied hydrocarbon gas can improve the operating conditions of the systems.
\end{abstract}

\section{Introduction}

For the energy system of the world, an industry of liquefied hydrocarbon gases (LHG) is dynamically developing. World consumption of liquefied hydrocarbon gas grows by $10 \%$ per year, and gas-air fuel only by $2.4 \%$. So, according to the forecasts of experts of the International Gas Union (IGU), the share of liquefied hydrocarbon gas by 2030 may be about $42 \%$ of total natural gas consumption [1].

Liquefied natural gas is a fairly promising energy carrier, which is well suited for gas supply to remote areas where the delivery of energy through pipelines is impossible (due to the specific geographical location) or unprofitable. Storage of liquefied hydrocarbon gas is carried out at cryogenic temperatures, therefore, compliance with temperature parameters has a direct impact on industrial safety. Depressurization of storage tanks of liquefied

\footnotetext{
Corresponding author: mani495@mail.ru
} 
hydrocarbon gas can lead to fires and explosions, therefore ensuring safety at its storage facilities is a key task [2].

Storage tanks of liquefied hydrocarbon gas can be divided into three main groups:

1. Stationary storage tanks. They are the part of the LHG delivery, gasification and storage system. They serve for long-term storage of liquefied hydrocarbon gas under a pressure of $0.2-6 \mathrm{~atm}$ [3]. The volume of reservoirs is more than $50 \mathrm{~m} 3$.

2. Transport storage tanks. They serve for the delivery of LHG to the consumer. The maximum pressure under which the product is located reaches $16 \mathrm{~atm}$.

3. Technological storage tanks. They are designed for LHG, which is produced at the complex, and carry out the function of providing consumers with liquefied gas according to a certain schedule. The volume of such reservoirs is less than $50 \mathrm{~m} 3$ [4].

An important aspect of ensuring the safe operation of LHG tanks is their supply with an automated safety management system that interconnects sensors (mainly pressure and temperature sensors), alarms and actuators (compressors, gate valves, etc.). This system should control the operation of the tank to prevent the following situations [5-6]:

1. Prevention of LNG overflow. Overflow control is carried out using level sensors. In case of an excess of the product level, it must be pumped out to the additional tank through the emergency valve. The design of such systems should be carried out by a special organization, whose employees have the appropriate qualifications.

2. Prevention of increase (or decrease) in pressure above (or below) the design value in the tank. The registration of pressure by the height of the tank allows one to identify its increase and, if exceeded, to ensure its discharge using vapor recovery valves. The decrease in pressure should be compensated by the supply of inert or natural gas to the tank. Argon can be used as inert, but due to its high cost, nitrogen is more often used.

3. Prevention of a rise in temperature of the tank by irrigation. The feed system located on the roof of the tank is switched on if the temperature rises by the signal of the temperature sensors (on the ferrule of the tank).

4. Prevention of the rollover development. It is recorded by temperature sensors. In the event of a strong heterogeneity in temperature readings, the gas supply system to another mixing tank is turned on. Prevention of the rollover development is also achieved through the use of a wide range of control systems based on mathematical models of various gas flows in the tank.

During the operation of gas consumption networks in public and administrative buildings, the following types of work must be ensured by the operating organizations, according to Russian State Standard GOST R 54961-2012.Gas distribution systems. Consumers gas networks. General requirements to operation:

1. put of gas consumption networks into operation;

2. maintenance and repair of external and internal gas pipelines;

3. maintenance and repair of household (gas stoves, water heaters, heat generators, convectors, etc.) and other (restaurant stoves, grills, mini-bakeries, etc.) gas-using equipment;

4. reconstruction of gas consumption networks with domestic gas-using equipment (if necessary);

5. emergency dispatch services;

6. decommissioning of gas pipelines and gas-using equipment.

Liquefied gases are flammable and explosive, have low toxicity, a specific characteristic odor, and are classified as substances of the 4th hazard class according to the degree of exposure to the body.

Liquefied gases form explosive mixtures with air at a concentration of propane steam from 2.3 to $9.5 \%$, of normal butane from 1.8 to $9.1 \%$ (by volume), at a pressure of $0.1 \mathrm{MPa}(1 \mathrm{~atm}$.$) and at a temperature of 15 \ldots 20^{\circ} \mathrm{C}$. 
The self-ignition temperature of propane in the air is $470{ }^{\circ} \mathrm{C}$, of normal butane $-405^{\circ} \mathrm{C}$. The maximum permissible concentration in the air of the working zone of saturated hydrocarbons (propane, normal butane) is $300 \mathrm{mg} / \mathrm{m}$, of unsaturated hydrocarbons (propylene, butylene) - $100 \mathrm{mg} / \mathrm{m}^{3}$, according to Russian State Standard GOST R520872003. Liquefied hydrocarbon fuel gases. When designing and operating plants, transporting and storing liquefied hydrocarbon gases, the properties of the individual components and their mixtures must be taken into account.

During transportation, storage or regasification, hydrocarbon gases are in the form of a two-phase liquid-steam system, i.e. consist of liquid and steam phases, according to Russian State Standard, National standard of the Russian Federation GOST R 59 961-2012 Gas distribution systems. Gas consumption networks.

The main properties of the steam phase that must be taken into account during operation are as follows:

1. high density;

2. slow diffusion;

3. low flash point;

4. inconsistent calorific value.

5. The main properties of the liquid phase are as follows:

6. strong volume compression and expansion;

7. low relative density;

8. cooling ability during regasification.

All these properties must be taken into account when operating liquefied hydrocarbon gas plants [7].

Liquefied hydrocarbon gases include hydrocarbons, which under normal conditions are in a gaseous state. At a relatively small increase in pressure they turn into a liquid state. It is believed that despite the technical condition of gas distribution networks, the amount of gas loss is unchanged and depends on the geometric volume of gas networks, pressure and leakage coefficients [8]. With a decrease in pressure, these hydrocarbon liquids estimate and pass into the steam phase. It allows transporting and storing liquefied hydrocarbons with the conveniences typical of liquids and controlling, regulating and burning gaseous hydrocarbons with conveniences for natural gases [9].

Liquefied gases are a high-quality fuel for the needs of food preparation, heating, for a number of technological processes in industry and agriculture.

The main components of liquefied hydrocarbon gases are propane and butane.

\section{Materials and methods}

Depending on the method of application, the following brands of liquefied gases are determined:

1. mixture of propane and butane, technical, winter;

2. mixture of propane and butane, technical, summer;

3. technical butane.

Liquefied gas in a sealed vessel exerts pressure on the walls of the vessel, called steam pressure, which is a temperature function. The more light hydrocarbons contained in the gas, the higher the steam pressure.

When designing and operating systems for supplying consumers with liquefied gas, it is necessary to know the thermodynamic processes that occur during the selection of the steam phase. 


\section{Results}

A liquid mixture of hydrocarbon gas under pressure enters the cylinder. The molar composition of the mixture is $\sum A=1$ (Figure 1).

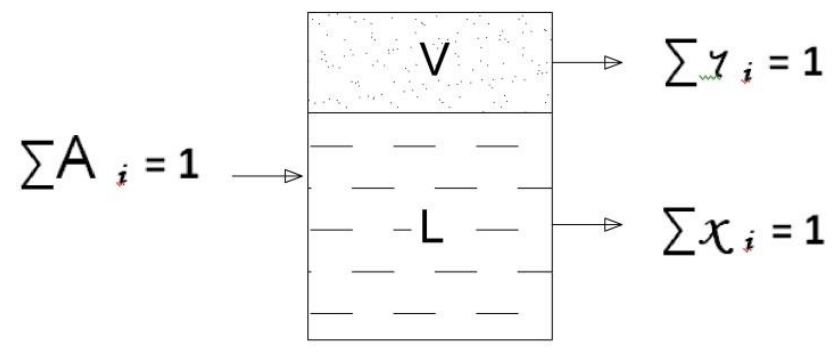

Fig. 1. The process of separating a hydrocarbon mixture into liquid and steam: $\mathrm{V}$ is the fraction of the total number of moles in the steam phase; $\mathrm{L}$ is the fraction of the total number of moles in the liquid phase (moreover, $\mathrm{V}+\mathrm{L}=1$ )

Dalton's law determines the pressure of gases' mixture that do not enter into chemical reactions between themselves. Each individual gas, called a component, behaves in the mixture as if there were no other gases in the volume. It occupies the entire volume and obeys the equation of state written for it. This equation determines its individual (partial) pressure. The mixture pressure $P_{c m}$ is equal to the sum of the partial pressures of the components:

$$
\mathrm{cm}=\sum P_{i}
$$

where $P_{i}$ is the partial pressure of the gas $i$, that is, the pressure that this gas would exert if it occupied the entire volume occupied by the mixture.

The partial volume $V_{i}$ of the $i$ th component is the volume occupied by it at the temperature and pressure of the mixture.

The composition of gas mixtures is set in molar fractions.

According to Raul's law, the partial steam pressure of a given component in a liquid mixture is equal to its molar concentration in the liquid, multiplied by the steam pressure of this component in its pure form at the temperature of the mixture:

$$
i=x_{i} \text { isat, }
$$

where $P_{i}$ is the partial pressure of the $i$ gas in the liquid mixture;

$x_{i}$ - molar fraction of $i$ component in the liquid mixture;

$P_{i \text { sat }}$ - steam pressure of pure component at mixture temperature.

From the equality of partial pressures, we obtain the basic equation

$$
\begin{aligned}
& \mathrm{r}_{\mathrm{i}} \mathrm{P}_{\mathrm{cm}}=\mathrm{x}_{\mathrm{i}} \mathrm{P}_{\mathrm{i} \text { sat }} \\
& \mathrm{A}_{\mathrm{i}}=\mathrm{r}_{\mathrm{i}} \mathrm{V}+\mathrm{x}_{\mathrm{i}} \mathrm{L},
\end{aligned}
$$

where $A \mathrm{i}$ is the total number of moles of $i$ component;

$r_{i} V$ is the number of moles of $i$ component in the steam phase;

$x_{i} L$ is the number of moles of $i$ component in the liquid phase.

Temperature $t$ and cylinder pressure $P$ are set. It is required to determine the composition of the steam $\sum r_{i}=1$ and the liquid phases $\sum x_{i}=1$.

The equation of material balance for the component: 


$$
\mathrm{A}_{\mathrm{i}}=\mathrm{r}_{\mathrm{i}} \mathrm{V}+\mathrm{X}_{\mathrm{i}} \mathrm{L}
$$

Put the value of $x_{i}$ :

$$
x_{i}=r_{i} \frac{P_{\mathrm{cm}}}{P_{\mathrm{sat} i}} .
$$

Then we obtain:

$$
A_{i}=r_{i} V+\frac{r_{i} P_{\mathrm{cm}}}{P_{\mathrm{sat} . i}}(1-V)
$$

Next we define:

$$
r_{i}=\frac{A_{i}}{V+\frac{P_{\mathrm{cm}}}{P_{\mathrm{sat} . i}}(1-V)}
$$

Summarize $r_{i}$ for all components:

$$
\sum r_{i}=\sum \frac{A_{i}}{V+\frac{P_{c m}}{P_{\text {sat } i}}(1-V)}=1
$$

From this equation we determine the value of $V$ and calculate the composition of the steam and liquid phases.

We can get the calculated dependence taking into account:

$$
r_{i}=x_{i} \frac{P_{i s a t}}{P_{\mathrm{cm}}}
$$

Than we define $A_{i}$ :

$$
\boldsymbol{A}_{\boldsymbol{i}}=\boldsymbol{x}_{\boldsymbol{i}} \frac{\boldsymbol{P}_{\text {isat }}}{\boldsymbol{P}_{\mathrm{cm}}}(1-L)+\boldsymbol{x}_{\boldsymbol{i}} L
$$

Summarize $x_{i}$ for all components:

$$
\sum x_{i}=\sum \frac{A_{i}}{L+\frac{P_{\mathrm{cm}}}{P_{\mathrm{sat} . i}}(1-L)} .
$$

From the given equation, we determine the value of $L$ and calculate the composition of the liquid and steam phases.

We determine the composition of the steam and liquid phases, if the initial composition of the mixture

$$
\begin{aligned}
& \mathrm{A}_{\mathrm{C}_{3} \mathrm{H}_{8}}=0.5 ; \\
& \mathrm{A}_{\mathrm{c}_{4} \mathrm{H}_{10}}=0.3 ; \\
& \mathrm{A}_{\mathrm{HC}_{4} \mathrm{H}_{10}}=0.2 .
\end{aligned}
$$

We determine the steam pressure of the mixture components at $\mathrm{t}=20^{\circ} \mathrm{C}$ in $\mathrm{MPa}(\mathrm{abs})$.

$$
\begin{aligned}
& \mathrm{c}_{3} \mathrm{H}_{8}=0.83 ; \\
& \mathrm{c}_{4} \mathrm{H}_{10}=0.306 ; \\
& \mathrm{Hc}_{4} \mathrm{H}_{10}=0.23 .
\end{aligned}
$$

Calculate the mixture pressure:

$$
0.5 \cdot 0.83+0.3 \cdot 0.306+0.2 \cdot 0.23=0,553 \mathrm{MPa} \text {. }
$$

Then we find the value $\sum x_{i}=1$ : 


$$
\begin{aligned}
& \sum x_{i}=\sum \frac{A_{i}}{V+\frac{P_{\mathrm{cm}}}{P_{\mathrm{sat} .} i}(1-V)}=\mathbf{1} \\
& x_{i}=\frac{0.5}{V+\frac{0.553}{0.83}(1-V)}+\frac{0.3}{V+\frac{0.553}{0.306}(1-V)}+\frac{0.2}{V+\frac{0.553}{0.23}(1-V)}=1 .
\end{aligned}
$$

Solving this expression, we obtain $V=0.49$.

Then we determine the composition of steam mixture:

$$
\begin{aligned}
& r_{\mathrm{C}_{3} \mathrm{H}_{8}}=\frac{0.5}{0.49+\frac{0.553}{0.83} 0.51}=\frac{0.5}{0.49+0.667 \cdot 0.51}=0.65 ; \\
& r_{\mathrm{C}_{4} \mathrm{H}_{10}}=\frac{0.3}{0.49+\frac{0.553}{0.306} 0.51}=\frac{0.3}{0.49+1,807 \cdot 0.51}=0.22 ; \\
& r_{\mathrm{HC}_{4} \mathrm{H}_{10}}=\frac{0.2}{0.49+\frac{0.553}{0.23} 0.51}=\frac{0.2}{0.49+2.4 \cdot 0.51}=0.13 .
\end{aligned}
$$

Then we determine the composition of liquid phase:

$$
\begin{gathered}
x_{i}=\frac{r_{i} P_{\mathrm{cm}}}{P_{i \mathrm{sat}}} ; \\
x_{\mathrm{c}_{3} \mathrm{H}_{8}}=\frac{0.65 \cdot 0.553}{0.83}=0.4 ; \\
x_{\mathrm{c}_{4} \mathrm{H}_{10}}=\frac{0.22 \cdot 0.553}{0.306}=0.3 ; \\
x_{\mathrm{HC}_{4} \mathrm{H}_{10}}=\frac{0.13 \cdot 0.553}{0.23}=0.3 .
\end{gathered}
$$

\section{Conclusions}

Based on the peculiarities of the system of gas distribution of populated areas, it is advisable to apply such a technical solution, in which gas supply to household consumers, boiler houses, municipal and agricultural enterprises is carried out from the distribution network of high and medium pressure through autonomous combined pressure regulators installed near high-rise buildings [10].

The gas supply scheme in these cases can be of two variants [11,12]:

- gas control point with gas measurement, where the pressure is reduced from 1.2 $\mathrm{MPa}$ to 0.3 or $0.6 \mathrm{MPa}$, a single-stage distribution network of medium or high pressure, combined pressure regulators with output low or medium pressure;

- single-stage distribution network with gas supply of $1.2 \mathrm{MPa}$, combined pressure regulators with medium output pressure and combined with them low pressure combined pressure regulators. This scheme makes the gas distribution system very flexible, independent of the connection of any object is achieved. It is possible to connect an additional load. At the same time, metal consumption in distribution networks decreases, due to the reduction in the diameter of the distribution gas pipeline.

The main basic element of the gas distribution scheme is a combined gas pressure regulator that performs the functions of a gas regulator: reducing the gas pressure to a predetermined level, automatically maintaining the gas pressure at a predetermined level regardless of changes in inlet pressure and gas flow, automatic gas shut-off in emergency rises or gas pressure drops after in excess of the setpoints [13].

Regasification of liquefied gases can be called regasification with natural evaporation, that is, when air and soil are used as heat carriers, significantly affect the natural evaporation of liquefied gases and other significant factors except the temperature regime. 
The evaporative capacity of cylinder and tank installations is reflected by the following main factors: thermal resistance of the protective insulation of the underground tank, the walls of cylinder and tank; thermal resistance of the soil, speed and humidity; cyclical selection of the steam phase; the degree of filling with the liquid phase of the tank; component composition of the liquid phase; thermal resistance to heat transfer from ambient air, the degree of contamination of the tank walls; thermal interaction of underground tanks $[14,15]$.

During the offtake of the steam phase from the cylinder, the equilibrium state between the liquid and gas is violated. The steam pressure of liquefied gases will gradually decrease. At the same time, the ratio of components is constantly changing: the relative content of lighter hydrocarbons will decrease, and the content of heavier ones will increase.

At maximum gas offtake from the cylinder, the temperature of liquefied gases will decrease due to the lack of heat supply from the outside. The more a liquefied gas evaporates in a cylinder during natural evaporation, the lower temperature will be under the indicated conditions.

With an intensive gas flow, the temperature of the liquid will decrease sharply, evaporation will be disrupted, and the vessel will freeze. This phenomenon is especially often observed during the operation of gas-balloon installations: with an increase in the offtake of liquefied gas steam from them. Evaporative capacity also depends on the degree of filling of the cylinder. The larger the wetted surface, the greater the evaporative capacity.

\section{References}

1. V.A. Voronov, Ya.V. Martynenko, Int. Res. J., 04 (58), 4 (2017)

2. E.V. Shevchuk, Int. Res. J, 11 (53), 141-143 (2016)

3. V.S. Kravnova, V.Ph. Martynuyk, Bull. Rus. St. Un. Oil. Gas., 1, 154-161 (2011)

4. G.R. Bazarov, S.S. Mirzaev, I. Gimranov, Sci. Techn. Educ., 2, 28-29 (2016)

5. G.G. Vasil'yev, S.G. Ivantsova, A.I. Rakhmanin, Gas ind., 11, 57-61 (2013)

6. V.A. Voronov, Ye.A. Lyubin, Ye.Yu. Zagorodneva, Scient. Techn. J., 759-767 (2015)

7. V.A. Zhila, Ye.B. Solovyeva, M.D. Gulyukin, Scient. Rev., 22, 27-32 (2016)

8. O. Gnezhdilova, A. Malysheva, A. Gagulina, A. Ovsienko, FORM-2019, 97 (2019)

9. V.A. Zhila, E.B. Solovyeva, Yu.G. Markevich. Development of a methodology for determining the optimum reliability indicators for elements of gas supply system (2016)

10. G.M. Kostrova, Requirements for industrial safety at gas distribution and gas consumption facilities (2016)

11. V.A. Zhila, E.B. Solovyeva. MATEC Web of Conferences, 170, 03016 (2018)

12. Yu.A. Tabunshchikov, V.I. Prokhorov, O.N. Bryukhanov, V.A. Zhila, A.K. Klochko, Vestnik MSBI, 4, 73-77 (2012)

13. Ye.B. Solovyeva (IJMET), 9, 2,761-764 (2018)

14. A.P. Usachev, A.Yu. Frolov, A.V. Rulev, T.A. Usacheva, A.S. Trushch, Sci. Techn. Probl. Improv. Develop. Gas Sup. Syst., 1, 84-99 (2007)

15. I.G. Kiseleov, M.Yu. Kudrin, V.G. Zalomin, Procee. St. Petersb. Univ. Rail.. 1(34), 90-96 (2013) 